\title{
VR/Urban: Spread.gun - Design Process and Challenges in Developing a Shared Encounter for Media Façades
}

\author{
Patrick Tobias Fischer \\ University of Strathclyde \\ 26 Richmond Street, Glasgow G1 1XH \\ fischer@cis.strath.ac.uk
}

\author{
Christian Zöllner \\ University of the Arts Berlin \\ Strasse des 17. Juni 118, 10623 Berlin \\ mail@christian-zoellner.com
}

\author{
Eva Hornecker \\ University of Strathclyde \\ 26 Richmond Street, Glasgow G1 1XH \\ eva.hornecker@cis.strath.ac.uk
}

\begin{abstract}
Designing novel interaction concepts for urban environments is not only a technical challenge in terms of scale, safety, portability and deployment, but also a challenge of designing for social configurations and spatial settings. To outline what it takes to create a consistent and interactive experience in urban space, we describe the concept and multidisciplinary design process of VR/ Urban's media intervention tool called Spread.gun, which was created for the Media Façade Festival 2008 in Berlin. Main design aims were the anticipation of urban space, situational system configuration and embodied interaction. This case study also reflects on the specific technical, organizational and infrastructural challenges encountered when developing media façade installations.
\end{abstract}

Media Façade, Urban Media Intervention, Mobile Tangible User Interface, Interface Design, Embodiment, Shared Encounters, Social Technology, Situated Technology.

\section{INTRODUCTION}

The urban environment has always been the design space of architects and urban planners. With the advent of media façades and urban screens a new type of design element has emerged. Our cities turn into dynamic and programmable surfaces [10]. Analogue billboards are substituted with digital ones and the character of buildings is boosted by a digital skin. The surface of the city is augmented with digital displays, sensors and ubiquitous structures such as wireless networks. The building structures of private properties are virtually extended and reach over their boundary line into public space and other properties. However, this type of digital extension is different from infrastructural elements such as electricity, heating, water, ventilation, etc. Most digital elements often solely serve commercial interests rather than people's needs. Media façades are thus often associated with "light polluting" devices, mass media giants or flickering advertisement messages. Most urban screens are used as a one-tomany communication device, taking the role of a big TV screen, rarely unfolding their architectural potential and integrity. When using a media façade as a design element for their building, most architects do not seem to invest much effort into considering what content it might host.

We believe that $\mathrm{HCl}$ should anticipate the trend of augmenting urban space with digital displays in socially useful ways (cf. the discussion about the role of values in $\mathrm{HCl}[8,11])$. We propose to focus on developing ways of replacing unidirectional with bidirectional communication in order to enhance social life, e.g. through digital shared encounters. Our conscious design decision is to support social values of city life, such as expressivity, public space, and serendipitous encounters with strangers. The term matching our design goals best is shared encounter [29]. A shared encounter is a computer supported social experience situated in public space that might cause social change.

In this paper, we discuss our Spread.gun installation as a case study exemplifying this approach. We will outline the concept, describe the design process and its multidisciplinary design aspects, and reflect on the specific technical, organizational and infrastructural challenges encountered when developing media façade installations, followed by preliminary findings.

\subsection{Background}

In the last few years, researchers and designers have begun to explore the new medium of media façades in various ways [3]. Some interactive realtime explorations have been done on the BBC Big Screens, which are spread throughout major cities in the UK. These include the BBC Public Space Broadcasting (PSB) project hosting Hand from Above by Chris O'Shea, Red Nose Dribbling by IDCD [19], Every passing Moment [28] and Hungry Hungry Eat Head by Hudson-Powell \& Joel Gethin Lewis. These projects demonstrate great potential as well as technological challenges in outdoor scenarios. In general, they explore the feasibility of interfaces with camera input, Bluetooth, and mobile phones in the context of urban screens. Another initiative supporting a variety of interactive shared encounters 
on media façades is the International Urban Screens Association (IUSA) [1], Miriam Struppek's scientific examination [27], and the Media Façade Festival [21] series, which involves several Media Labs across Europe.

Most of the above mentioned projects remain research and technology probes that explore the possibilities of interaction between user and urban screen with existing input devices and lack convergence of architecture and content, and a relation between user and architecture. On the other hand, there are already architectural concepts that demonstrate how architecture can integrate digital content in an aesthetical way. For example, the Federation Square in Melbourne has an agoralike plaza in front of its media façade. This allows observers to linger and gives them an opportunity to start conversations with strangers. Another example of coherent architecture and digital content is the Giga Center in Cologne at Rudolf Plaza. The building embeds a $2.7 \mathrm{~m} \times 13.5 \mathrm{~m}$ projection area across four levels. The unusual format does not adhere to commercial standards and thus depends on custom-made content. Most of the content shown is created by artists or students of the Academy of Media Arts (khm). Both examples show how cultural and social value may be generated. Nevertheless, as architects focus on the relations between people and architectural space (e.g. buildings) and more recently on the relationship between content and architectural space, we suggest that $\mathrm{HCl}$ researchers should anticipate these dimensions to create meaningful and consistent shared encounters.

Media façade projects are an interdisciplinary endeavour that requires design sensibility and a significant amount of software development. Designers and Software Engineers tend to have very different working styles and approaches, and this can initially create friction. Yet what seems like a chaotic process or 'black art' has its own rigour, and designerly approaches are slowly making their way into $\mathrm{HCl}[16,30]$. We believe that a designerly approach is essential for developing successful media façades, because the emotional and aesthetic aspects of the user experience are at least of the same importance as functionality or usability. Furthermore, there are only few 'good' examples of media façades so far and urban space is a new context for $\mathrm{HCl}$. Media façade development thus has all of the characteristics of a design process: uncertainty, particularity, and exploration of an openended problem [16].

$\mathrm{HCl}$ researchers have only recently begun to explore this domain $[3,4,19,23,25,28]$ which poses very specific technical and infrastructural challenges that so far have been rarely reported on. In general, there is only sparse literature sharing practical experience and strategies for implementing novel technologies in outdoor settings. As Harris et al [12] note, many aspects that are typically taken for granted, such as network coverage, electricity etc. suddenly become an issue when developing for an outdoor setting. Discussing such challenges encountered in our project and how we resolved them is thus part of our contribution.

\section{INITIAL SITUATION}

In 2007 the curators of the Media Façade Festival (MFF08) called for artists living in Berlin to participate in creating artworks for media façades. One of the authors (P. T. Fischer) asked friends in $\mathrm{HCl}$ research, software development, design research and visual arts to participate, and thus the collective VR/Urban was born. The initial proposal was developed for the SAP façade. Later in the design process the installation was moved to the media façade of the Collegium Hungaricum Building (CHB). The success of the resulting "artwork" Spread.gun motivated us to reflect on the design process of what we now call 'shared encounter'.

\subsection{From Inspiration to Vision}

The core concept of the Spread.gun was inspired by graffiti culture, which is practiced by people that know a lot about their city environment and relate directly to it. Graffiti artists have developed a variety of methods to 'use' the city, communicate through it, or reclaim space in the city. They have a tightly coupled relation to their medium of expression (the built environment). They rely on their body and physical abilities, fuelled with adrenaline, when roaming through the nightly city in order to finish their artwork. They don't finish it just anywhere, but in the chosen location that supports the work and the message. Location is indeed an aspect of graffiti that is crucial to meaning. Some graffiti artists have a very delicate and distinct perception of architectural space and connect their artwork very tightly to the build environment (examples can be found on ecosystem.org). However, doing graffiti has a variety of motives which have been analyzed in [22]. It is a deeply emotional and satisfying way of expression, which might be politically motivated, for the purpose of increasing personal fame (social capital), or for creative expression. Extracting some of these experiences of graffiti "adventures" and reinterpreting them was one of our motivations.

During the conceptual phase of the installation we were fascinated by a specific form of graffiti that uses plastic bags filled with liquid paint, which are thrown against buildings for various reasons. The level of aesthetics reached by the simple means of filling a bag and throwing it is really amazing. Even without any skill of drawing or calligraphy, one is able 
to express oneself, manifested in a coloured splat. The feeling of uncertainty while doing it, of how the splat will look when it has hit the wall, adds to the emotional value of this expressive act. Moreover, it is a very personal way of expression, because the act of throwing is done with one's own hands and not by using a tool that needs to be mastered first. Thus, the project's vision was born; to invent a digital version of a coloured splat. Everything that followed added to the idea, experience and magic of throwing coloured bags onto a wall. But for our project the wall would be a digital media façade and thus the colour bags would be virtual.

\subsection{Conceptual Phase}

In contrast to conventional media installations, which are usually placed indoors, ours had to be situated in urban space. Thus, some considerations had to be made. How can throwing a digital colour bag onto a media façade have a similar emotional effect as if you would do it in real life? How can we preserve the magic and unpredictability of the real experience? What should a virtual colour bag look like? The installation itself should be pleasurable, humane, non-violent, non-digital and non-anonymous.
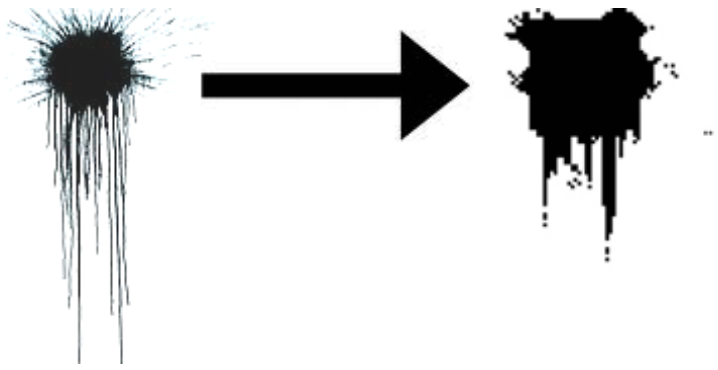

Fig. 1 Aesthetic result of minimum action

In addition to these questions and requirements we identified basic indicators for a failure of our design concept. We did not want people to just have a look at the installation, figure out how it works, and then walk away. We did not want the installation to be deconstructed by the visitor, or the technology to be in the foreground of the experience. There should be a very simple explanation for how the installation works: One can throw virtual stuff on façades - full stop. For the experience we aimed to create, it was much more important to have everything emerging out of the designed situation, than seeing and understanding the technology and how it works.

Another core idea was to channel people trough several stages of interaction [7, 20]. Passersby should become observers, performers and participants. Also performers' interaction should be comprehensible for observers so as to attract new participants. To design for watching and learning would also make the installation self-explanatory and self-regulating. This became an important aspect, because we wanted to allow participants to add personal messages to their splats. This means that not only a colour bag is thrown onto the façade, but also a more or less meaningful text statement. Thus, it was important that observers understand that the messages are typed by a performer who then shoots the message. This way, observers are also given time to think of a potential message they would like to shoot.

Apart from the users and us creators, the owners of media facades need to be thought of as a stakeholder, especially when allowing user input in the form of text. This is because uncontrolled content can be sent to the façade and the question will be asked who is responsible for un-censored content. In fact, only very few installations do not censor content. To evade this discussion, we argued to sponsors and façade owners that due to the ability to associate the author of a message with a message-splat, established social norms of urban environments apply. Throwing a virtual message would be a very visible activity and thus not anonymous so that social norms would apply. Lawrence Lessig [15] simplified the regulating instances of social life to four aspects: Law, architecture, norms and code. All four aspects regulate the situation in front of our public media screen. Law prevents people from posting e.g. nationalistic offences, architecture (and the installation setup) creates an association between author and message, and norms regulate the use of the installation, leaving code and the interfaces for us to design a situation of uncensored free speech and user experience without violating public life.

\subsection{Designing for spatial settings}

Graffiti is inextricably situated in urban space. When designing an interactive system, the spatial setting matters in the same way for the system designer as it does for graffiti artist, and thus should be taken into account during the design and analysis process. For an even better design, the situation should be considered. McCullogh [17] distinguishes between setting and situation: Setting is the ad hoc configuration of the environment, and situation is the much richer term that takes pedestrian flow, social configuration, history and cultural behaviour into account. The situation should be taken as preset, which the media intervention or shared encounter is designed upon. This widens the conventional design space in $\mathrm{HCl}$ and creates an even higher amount of complexity, which is discussed in [4] to provide a framework for these kind of installations. 


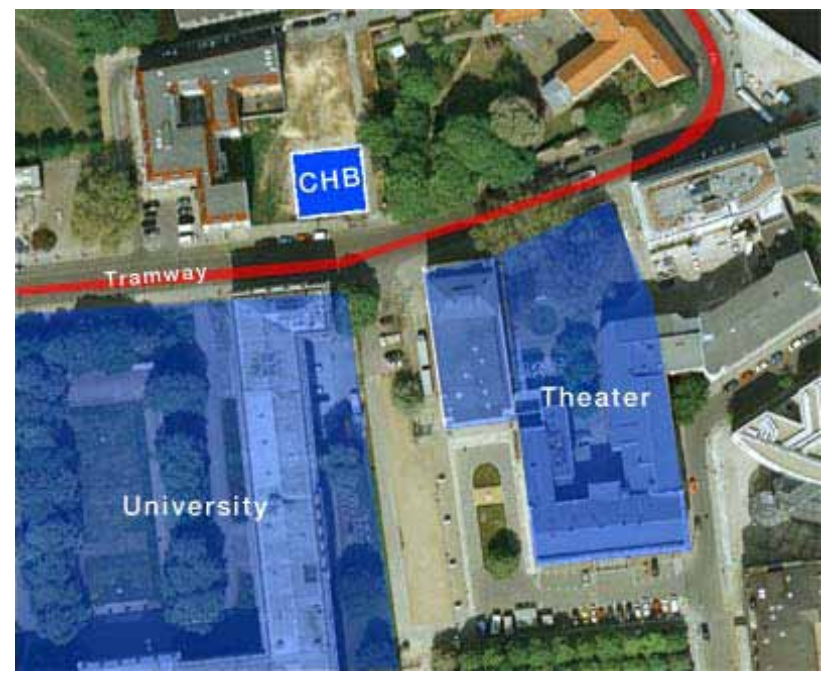

Fig. 2 Spatial overview of location around CHB

However, our aim was not so much to create an event or spectacle that radically modifies the actual situation, but an intervention that is used by some passers-by and to let other people just watch and pass without bothering. It should create an opportunistic encounter rather than create a strong expectation for the visitor.

For the Media Façade Festival 2008, the available façade was embedded in the Collegium Hungaricum Building (CHB) in Berlin-Mitte. Figure 2 shows the location of the building (see Fig.6) and important information about the setting. North of and behind the $\mathrm{CHB}$ is a natural edge in form of a canal, which only allows people to access and view the building from the east, south and west. Precise pedestrian flows were not available during the design process. Later-on we realized that the function of nearby buildings should be considered, as they dictate what sort of people will mainly find their way into the area. At daylight most pedestrians were students, because of the nearby University. At night, a lot of theatre visitors went by and briefly visited the Spread.gun. This might have an effect on audience expectations and perceptions. The location often also impacts on the actual design because it reconfigures the spatial setup. During the production process we had to move the exhibition place from another building to $\mathrm{CHB}$, and in consequence the whole concept changed. It seems that the meaning of place [13] is hard to ignore. The illustration below (Fig. 3) shows the final setting of the installation. In front of the projection, slightly off the middle of the screen at about $10 \mathrm{~m}$ distance the Spread.gun was mounted on an empty oil barrel sitting near the City Terminal from Wall AG (Fig. 7). Fortunately, the street in south direction was a dead end with only sparse car traffic (or only private traffic), which provided a large space for observers.

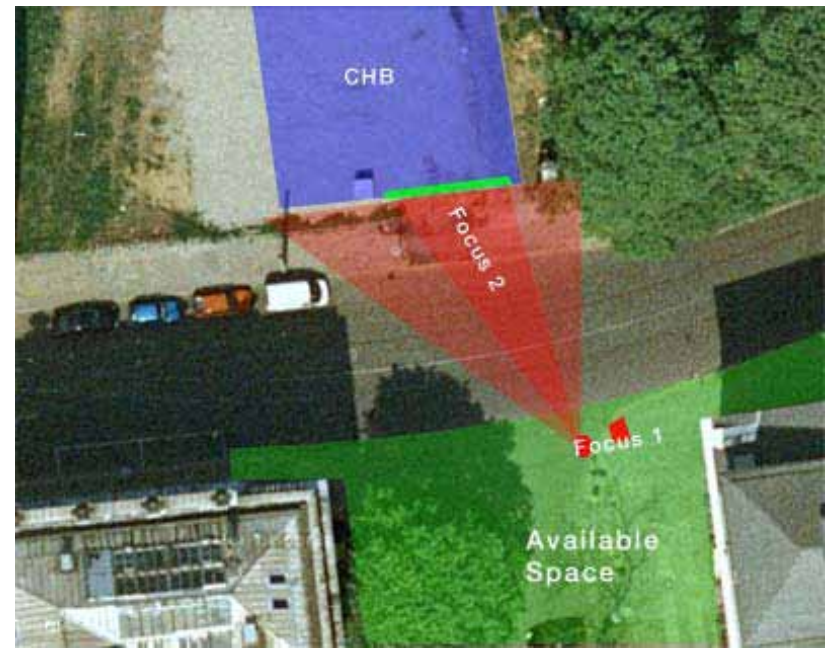

Fig. 3 CHB Setting

\section{DESIGN OBJECTIVES}

So far we described two key elements of the design process. We discussed how graffiti culture inspired us and how our design vision evolved from this. Second, we tried to understand the situation that might naturally occur in front of the building and envisioned future situations that might be created through our interactive installation. We have not yet described the Spread.gun itself. This mirrors the actual design process. There was a long period of design research about various ways to simulate throwing a colour bag. The Spread.gun was the result of this process.

For the experience of throwing a virtual colour bag on a façade, we knew that embodiment was crucial. With embodiment we here refer to large-scale physical bodily action. Body movement has been shown to increase engagement and to enhance social experience $[2,18]$. Pushing a tiny little button to transfer a message anonymously from e.g., a mobile to a screen would very likely result in a very different experience from what we aimed for. We also did not want to "freeze" people in place, something that sometimes happens when switching attentionfocus from the real world to the virtual world (see [6]). Furthermore, in our opinion a consistent installation cannot be created unless real and virtual space fuses into one experience. Embodiment was crucial for designing the situation and thus the action that had to be performed with the device should trigger and shape the feelings and emotions of the user.

A second effect we had in mind was to have people drift away from their original intention and to have a moment of personal enlightenment or dérive in the sense of Guy Debord's theorized concept [5]: Let them read the messages on the screen. Let them go or stay. Let them watch what is happening. Think about it. Perhaps an own statement comes to mind. Perhaps someone steps into the performance space 
and switches role from observer to being a short time performer and participant, finally leaving the stage. The provided stage with its roles is an opportunity for a dérive not a call for participation.

\subsection{Design of the Spread.gun}

The Spread.gun was our final solution for throwing colour bags onto the media façade. Its appearance was chosen carefully. Since the image of a gun is associated with violence and death, we had to search for a version that would not seem as harmful as modern weapons and provide a more romantic image. As we wanted to retain the aspects of power and force, we stuck to the overall metaphor. We felt ancient cannons to be less harmful than modern weapons and adopted a similar shape, giving it a modern touch through the possibility to assemble and disassemble it like a sniper weapon that one might know from action movies that after the assassination shot is taken apart and disappears in a violin case. The Spread.gun was thus made of plywood parts which allow for assembling it like a jigsaw and disassembling it again.

With the design decision to use the metaphor of a cannon, a rather profane problem arose. Shooting cannons is a rather digital action, similar to pressing a button and thus did not support our design goal of embodiment. Our version stripped away a lot of the actual physical effects of cannons. Imagine the noise, air pressure and the vibration a cannon like the one in Fig. 4 would create.

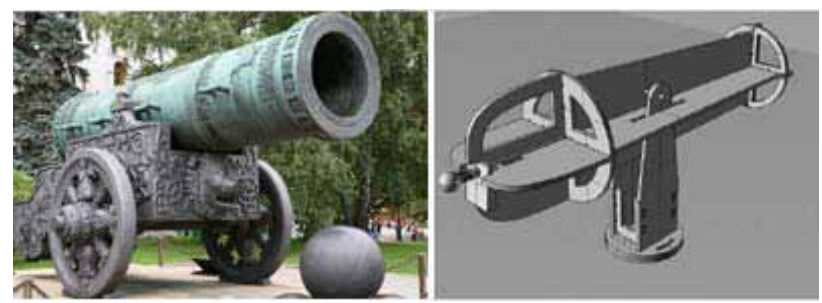

Fig. 4 Extracting affordances

Thinking about haptic feedback, we arrived at the solution of using a pinball machine trigger for three reasons. The pinball trigger would modulate the threatening shape of a cannon by adding a portion of humour. In addition, this provides the user with two types of feedback. A spring makes the user feel they are transferring some of their energy into the device, which then is released by a sudden jolt when the trigger is let go off. The directionality of the trigger movement also gives a cue about what is happening. It can easily be interpreted as launching something in the direction of the screen.

\section{TECHNOLOGY COMPOSITION}

Even though the project was initiated because we were given the opportunity to do something on a huge screen, the production process was mainly design driven. Technology was only used to implement the concept's vision and not for the sake of using a great technological feature. This section describes the technology setup we created to fulfil our vision.

4.1 Requirements Analysis

In contrast to purely software based projects, this one had a much higher complexity. The spatial integration into an urban environment resulted in a distributed system with a lot of software interfaces that created a high risk for the whole experience to fail.

The Spread.gun setup had a PC installed in front of the media façade, embedded in a City Terminal from Wall AG (Fig. 7). It had a 14" touch screen for entering messages, a one button trackball and a webcam. The latter two were not used, although the trackball was useful for the start-up procedure. Another PC functioned as server and was placed within the Collegium Hungaricum Building to do the rendering for the façade. The Spread.gun itself had a built-in microcontroller to send user input via USB cable to the touch screen terminal. An initial concept included two Spread.guns to get a fight of words started, but at the time only one Wall City terminal was available.

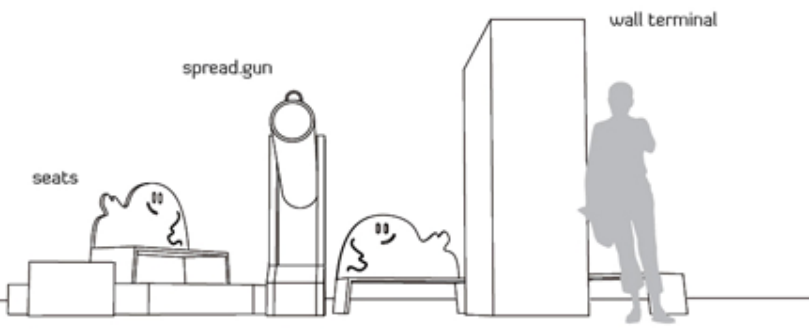

Fig. 5 Spread.gun setup

To shoot a message onto the façade, the user enters a message into the touch screen terminal via a virtual keyboard and "loads" it to the Spread. gun. To strengthen the connection between the user and the personal message, the user should also be able to select a personal colour for their splat. To indicate that the cannon is loaded with a message, a green light turns on near the pinball trigger. $X$ and $Y$ coordinates are recorded by the gun and sent to the client PC, which forwards them to the rendering PC. The colour splat should also have a physical behaviour in the visualization, to couple reality and virtuality tightly. Thus, a phase of flight and a phase of impact were modelled. In the first phase a colour bag shaped geometry flies in a generated trajectory to the point at which it is aimed. In the second phase little splashes splatter over the screen to indicate the impact, followed by dropping and flowing paint as if gravity is doing its work. The extension of real world physical behaviour into the virtual world was our design decision to support imagination and immersion of the user during interaction. 


\subsection{System Design and Implementation}

During the production process there were some concerns about the tramway (see Fig. 2) that crosses the setting midway between Spread.gun and media façade. The railway track did not allow us to connect both PC's with a LAN cable. Wi-Fi was not an option either, as prior experience showed that electrical interference from trams results in losing the Wi-Fi connection, and this is not acceptable for a real-time application. The solution initially embarked on was to use a directed wireless connection. Later in the deployment and testing phase we were told by technical support of the MFF08, that it was not possible to get these special devices for rent, which almost doomed our project to failure. Finally we decided to use a standard internet connection and at the end used a GSM USB-Stick which gives you internet wherever you are. Surprisingly the connection was fast enough to provide the impression of a real-time application.

However, implementtation of interactive systems for a media façade requires a diversity of skills in terms of network programming, adaptation of custom hardware and flexible software architecture. When dealing with media façades, in most

cases the setup of the hardware is highly customized and created by an external agency. For this reason, very often no one knows how it actually works, even the owner does not. When working with SAP (the original planned site for our installation) before $\mathrm{CHB}$, we only found one person with the key to the admin room and a crude handbook. These are bad conditions for creating custom-made distributed software, which will be rendered across multiple screens and projection walls. Fortunately, we were able to switch location to a more manageable back projection screen.

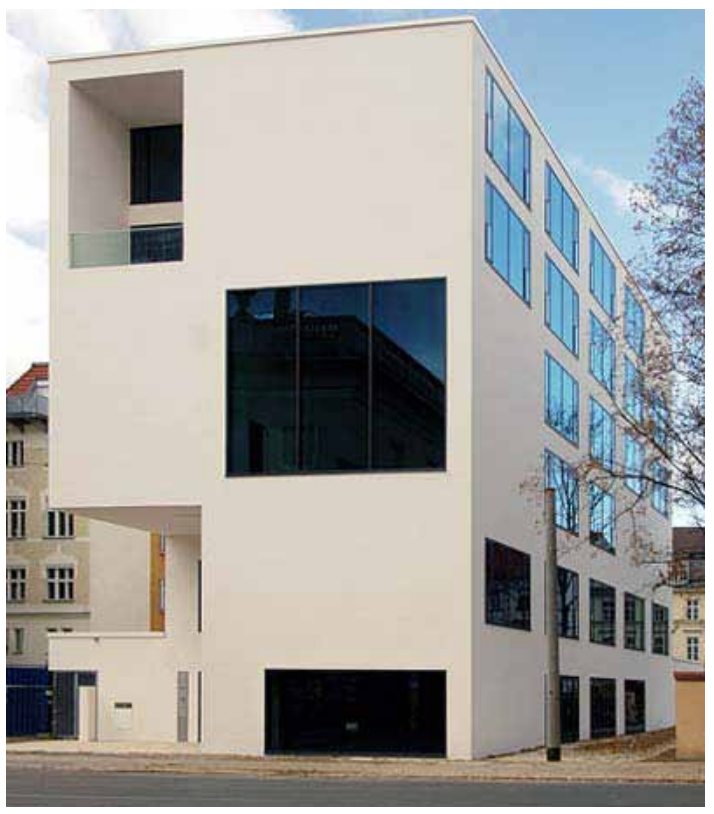

Fig. 6 CHB front with panorama projection window

\subsection{Hardware}

Shared encounters in urban spaces gain momentum through a great extent by scale and the built environment itself. The $\mathrm{CHB}$ is a distinctive architectural piece and combines Bauhaus style with modern technologies, such as a $40 \mathrm{~m} 2$ sized rear projection screen using a built in 15.000 ANSI Lumen projector (Christie LX 1500).

Fig. 6 shows the panorama window nicely fitted into the overall architecture of the building. The window can be used as a projection wall for internal use as well for external. Across the street the minimalistic and functional City Terminal was placed, $2304.5 \mathrm{~mm}$ high, $666.6 \mathrm{~mm}$ wide and $1498 \mathrm{~mm}$ long, a weight of $371 \mathrm{~kg}$, and designed by GK Sekkei, Japan.

As software developer or interaction designer one is seldom confronted with issues such as safety concerns. But especially when creating artefacts for outdoor use one will experience these again and again. For example, the terminal was installed specifically for the festival. We thus thought that we could choose its position as needed. Yet a weight of nearly $400 \mathrm{~kg}$ meant that the terminal becomes a safety issue when moved or altered. Thus, each modification request had to be done by service staff, which in return raised costs.

\subsection{Software}

The software system for the Spread.gun consisted, as mentioned above, of three main components: The client PC, rendering $P C$ and microprocessor software for the Spread.gun itself. The GUI of the touch screen terminal was created as an Adobe Flash application and consisted basically of a virtual keypad for composing a message and a colour picker. To forward the user input from the Spread.gun to the façade, an Arduino board with Atmel microcontroller sent appropriate information over USB serial connection to the Flash application. Because Flash does not allow serial input, a serial proxy was added to connect the Arduino and the Flash application. The connection between Spread.gun and terminal was bidirectional, which also allowed visual light feedback about the status of the Spread.gun. E.g. a green light indicated a loaded cannon and a red light an empty one. To propagate user input from the client PC to the rendering PC inside the building, the input was transformed to OSC packages. A hidden admin interface on the terminal turned out to be a useful feature. It allowed to calibrate the Spread.gun and to change the IP addresses, and enabled easy setup without a keyboard. The rendering application was coded in Java using the processing library [9] among others, like packages for OSC support or physics engines. 


\subsection{Test and Deployment}

When developing software for a broad audience, excessive testing is crucial. A lot of functional testing can be done in the lab, but many usability and functional problems only become visible when deploying in the wild [12]. The test phase was planned in three test screenings that also functioned as proof of concept for the funding partners. The client PC in the terminal was shared by six different artists that ran their own applications during the Media Façade Festival. Surprisingly this went very well even though we were concerned about missing files as result of multiple user access. A huge amount of software and drivers were installed in order to run all these diverse artworks. Sometimes this might result in incompatibility or crashes. But again, we were lucky. The most important software tool for installations like this turned out to be the remote desktop view. We used UltraVNC for configurations and program starts. In this way we were able to start up the installation without even needing to be in the administration room of the building. We also used it for remote testing if we had changed major parts of the software in the lab.

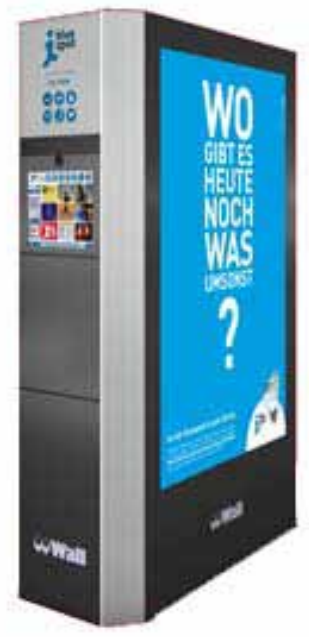

Fig. 7 Wall AG City Terminal

During testing we also realized other problems that cannot be anticipated when developing in the lab. For example, the font we used could hardly be read from a distance of $15 \mathrm{~m}$. Increasing the font size seems obvious but minimizes the possible amount of massages that can be shown on the screen. We solved this problem through an extensive search for more appropriate font types. Also, colour contrasts render differently on projections than on LCD or LED, again resulting in bad readability. It turned out that a black font is just as bad as a white font if users can select their own background colour. If they pick a dark colour, black fonts are hard to read, and if they choose light colours, white is a bad choice. In the end, this problem was solved only partially by simply drawing a black shadow behind the white characters.

\section{REFLECTION AND DISCUSSION}

In this section we will describe a possible way to minimize risk in a synchronized soft-, hardware and product production process and give recommendations to designing robust systems. We also describe how our shared encounter performed in situ and what effects on observers, performers and participants were observed.

\subsection{Production Process}

"If it does not work, you failed!" might be an obvious statement for software developers, but strangely enough this seems not apparent for product designers. During this interdisciplinary project, the differences in the roles of a designer and a software developer became noticeable. Each discipline uses very different processes during production. The design production process is highly incremental and results are always preliminary, because the final result is usually unknown at the beginning. The solution space is explored via several sketches, foam or cardboard models before more precise CAD models consolidate the final result. In engineering disciplines the result is clear from the beginning.

However, the outcome or final product also varies a lot. A product designer's design object is fix and demonstrates his hard work through manifestation in physical space. It cannot fail in the same way that an immaterial product can. If a software product does not work because of an infrastructural problem (e.g. internet connection is down) nothing can be shown. This makes real-time media installations in the wild fragile and fraught with risk. It also shows the deeper complexity between software products and physical products. Dynamic actions, dynamic memory, communication protocols, processing time are all properties of flow, which is much harder to design in contrast to things that are fixed. This makes a product designer a master of fixity in a flexible process and an interaction or software designer a creator of flow using a rather fixed and pre-planned production process.

During the physical production phase of the Spread. gun we experienced that a first design prototype always has bugs, no matter how long one thinks in front of the CAD software. It is not just that you might have forgotten to design a certain hole; also the properties of the tools one uses (e.g., a milling machine) are mostly unknown at the start. For example, we had no precise information about how thin a certain part of a material can be milled without having it destroyed by the machine itself. However, once you have your first prototype with these bugs, you might already be able to use it if you fix these bugs by hand. For example, the pinball trigger mounting of the Spread.gun had to be modified by hand to fit. Then we already built in the electronics to use it for some of our first test screenings. This turns 
the first prototype into a sort of test system, which is not perfect, but works. The next iteration generates a new prototype that will have fewer bugs and can be used as a development system at that time. Thus it is yet not fully functioning, because some parts might be missing. It cannot be used for testing, but it will be closer to the perfect result in contrast to the already existing test system. What we propose when doing interdisciplinary projects like these, is to adapt the production process to the best practice in agile system development and to have three systems in total: production system, test system and development system. This will triple the cost of hardware, but will give you the confidence, that:

- There is always a working system at hand the production system. No risk of total failure.

- There will be a better version shortly available (test system), after testing it. The testing can be done by others and issues found can be remedied straight away for the development system.

- The perfect system is under development, where fast changes can be made easily.

Having three systems can also help to do the product design process and system design process at the same time and minimizes the risk of total failure.

\subsection{Lessons learned on technology and infrastructure}

Creating interfaces for outdoor use poses a particular demand for robustness and safety, which even for a business like Wall AG is not a trivial task to design for. For instance, the touch screen of the City Terminal becomes unreliable if the temperature drops below $0^{\circ} \mathrm{C}$, although specification papers say different.

The complexity in this project was generated trough the three different subsystems of Arduino sketch, Adobe Flash GUI including Action Script and Processing renderings on three different hardware systems: Spread.gun, City Terminal and rendering PC. The latter furthermore have to communicate on different connection channels. In the end, four different programming languages, five applications and two transfer-protocols and channels where used to drive the installation. This heterogeneity requires a broad spectrum of knowledge and increases the risk of failure. It poses difficulties for technical staff to run the system when its creators are not at the site. Thus we propose to keep the amount of subsystems as low as possible and to aim for a homogeneous system structure.

This heterogeneity seems to have been a result of our incremental design process. On the one hand this allows the installation to be developed in a very flexible way; on the other hand it increases the complexity of the system and thereby creates a risk as mentioned above. But there were also situations where there was not enough flexibility, e.g., the Processing library is very good for rapid visualization tasks, but also limits flexibility for advanced software development, especially when the visual design is not widget based and highly custom. Here we come to the conclusion to choose the tools according to the team's skills and design goals.

In terms of media façade infrastructure and support, we recommend to always take account of and prepare for the worst case. Usually there will be no-one who can help with technical issues and the system administrators often fail to understand why these are vital for the installation. It is important to emphasize that technical needs are crucial for functioning. You may delegate, but this has to be done precisely.

\subsection{The Effects of the Urban Environment and Situations}

As outlined in the earlier section about spatial settings, the installation was designed for pedestrians passing by one after the other distributed over time. Unfortunately a different situation emerged as the festival opened its doors. At MFF08 at one point about 70 people came out of a bus and encountered something that can only be experienced in a rather slow way. The installation is not producing an immediate 'wow'-effect to a large audience. Even worse, it gets jammed by a mass of people. At this specific situation it was jammed, because one person had to step out of the group and act as performer. This puts a lot of pressure on this person, the more the larger the audience. Fortunately an official representative of the festival was keen to demonstrate. During the festival itself our passer-by design seemed to work, because we had exactly the situation we had designed for. This story shows that a design for a specific situation should not be misused for another situation. Two other exhibitions where we were persuaded to demonstrate the installation inside a building showed that this does not foster the same user experience as in an urban situation. It seems that besides of embodied interaction, scale and architectural context add to the user experience and overall momentum.

Another aspect of the effects of urban space on the installation arises from the meaning of place and its history. During the project we experienced how a location change from the SAP media façade to the $\mathrm{CHB}$ media façade changed the whole concept and statement. Positioning the Spread.gun in front of the SAP building would have risked that the CEO might veto the installation to avoid bad press. This might seem counterintuitive, but Berlin is a very political city with numerous leftist and anticapitalist activist groups. These might interpret the installation as a 
request for riot against SAP, which in Berlin is often seen as symbolizing capitalism, - in fact, the building has already been the target of several attacks. This is another example for how location creates context, altering the meaning of an installation.

A third aspect worth mentioning is that established social norms seemed to be working. The presence of observers and the visibility of interaction prevented performers from posting any inappropriate or unwanted messages such as those described in [24] where most messages contained sexual references. This example shows clearly that identity in contrast to anonymity helps to create some social control over content.

\subsection{Physicality, Emotion, and Engagement}

Embodiment, here interpreted as physical bodily action, seems to be able to create deep emotional effects. People described it as very satisfying to shoot a message to a screen in this way. On a very cold day the potentiometers in the Spread.gun did not deliver stable values for the crosshair on the screen. The crosshair was unusual to steer and a person described it like the gun seemed a little stubborn. But she liked the character of the gun which gave it an identity. She also thought it was done on purpose. We further learned that the physical form of the Spread.gun was much more appealing to the people than the City Terminal it was connected to. A lot of visitors approached the gun first, and as a result did not understand that they first had to load the gun by typing a messing into the terminal.

The appeal of reclaiming part of a building, leaving a trace, and making one's own use of it, also seemed to add to people liking the installation. Occupying space in urban environment is an often seen phenomenon. Some older men might take over a corner of the park for playing boccia or chess. The occupied place becomes part of one's life and an emotional relationship emerges. In terms of the $\mathrm{CHB}$ installation this phenomenon is worth to promote. The institute's agenda is to provide an open place for cultural events like art exhibitions or workshop. Aspects like allowing the public to reclaim a part of the building work well with this agenda.

Similarly, as reported by the creators of uPoi [26], with our media installation the participant's emotional affect seemed to be higher than the observers' affect. But different from uPoi this was not caused by the lack of understanding the connection between performer's interaction and the content on screen. The association between message and author can be inferred from the gesture of shooting something towards the façade. However, it is questionable whether it makes sense to design for equal emotional affect, because then the suspense curve that a passer-by goes through (from approaching the installation, watching others, collecting the courage or motivation to step up and use the Spread.gun) would be lost.

We further observed social interaction effects like the Honey Pot Effect [14], which can draw new observers towards the installation when seeing other people interacting. Being able to watch and understand the interaction furthermore enables people standing nearby to teach each other the use of the artefacts. In our case, observers even seemed to be able to learn from watching at a distance. It also turned out that most, if not all users were really ambitious about the proper appearance of their message on the façade. Position, colour and amount of messages were carefully chosen. People retyped their message if the splat was accidentally truncated or shot at a position where it could hardly be read. They also tended to aim where no other splats were or near them, but usually never on top of a prior message. This shows that a shot message has a value that is wanted to retain, and that people respect the 'ownership' of previous users for their messages.

\subsection{Cognitive "Error" Compensation}

As described earlier, interaction with the Spread. gun was divided in three steps. First, one enters a message at the touch screen terminal to "load" the cannon. Second, one aims and receives feedback from a little red crosshair on the screen that shows where the splat will go. Third, one pulls the pinball trigger and launches the message. During the design process we were concerned about the accuracy of the absolute coordinate system for the $x / y$ coordinates used for the virtual crosshair. We aimed for maximum horizontal and vertical input movement with the Spread.gun in order to maximize engagement with the artefact. On the contrary, it turned out that a maximum moving angle of $90^{\circ}$ horizontal and $70^{\circ}$ vertically would result in a huge target space depending on the distance to the screen. The screen available to us was $7,10 \mathrm{~m}$ $x 5,70 \mathrm{~m}$, which means that already at a distance of $10 \mathrm{~m}$ the turning angle needed would only be $-40^{\circ}$. At this point we decided not to mimic the realistic ballistic behaviour of the trajectory of a colour bag, but to maximize the physical movement required. This means that if someone turned the gun to the leftmost, then the crosshair would also be moved to the leftmost, ignoring the fact that the cannon was actually aiming way off the screen. Surprisingly none of our users realized the offset between real and virtual realm.

\section{CONCLUSION}

In this paper we reflected on the design process of a media installation situated in the urban environment. Inspired by established forms of graffiti culture, the 
multidisciplinary team developed a strong vision that created passion and ambition for each of VR/Urban's team members. We learnt that it is very difficult for the different production processes of product designer and software developer to run in parallel, while the media installation design is still in flux. It turned out that our incremental design approach resulted in a heterogeneous system structure with an inherent risk of total failure. To minimize risks for future developments, we propose adopting best practices from agile software development. Our installation Spread.gun is an example for new directions in $\mathrm{HCl}$, where a third discipline has been integrated -the architectural space. If $\mathrm{HCl}$ wants to understand shared encounters, the relation between human and space as well as content and space needs further exploration.

\section{REFERENCES}

[1] International Urban Screens Association. http:// www.urbanscreensassoc.org.

[2] N. Bianchi-Berthouze, W. W. Kim and D. Patel, Does Body Movement Engage You More in Digital Game Play? and Why? in Proceedings of int. conference on Affective Computing and Intelligent Interaction, 2007, Springer-Verlag.

[3] M. Brynskov, P. Dalsgaard, T. Ebsen, J. Fritsch, K. Halskov and R. Nielsen. Staging Urban Interactions with Media Facades. in Proceedings of INTERACT 2009, Springer, Heidelberg, 2009, 154-167.

[4] P. Dalsgaard, K. Halskov and R. Nielsen, Towards a Design Space Explorer for Media Facades. in Proceedings of OzCHI'08, 2008, ACM.

[5] G. Debord Society of the Spectacle. Rebel Press, 1992.

[6] E. Eriksson, T. R. Hansen and A. Lykke-Olesen. Reclaiming public space: designing for public interaction with private devices Proceedings of TEl'07, ACM, 2007.

[7] M. Finke, A. Tang, R. Leung and M. Blackstock, Lessons learned: game design for large public displays. in DIMEA 2008, 2008, ACM.

[8] B. Friedman Value-sensitive design. interactions, 3 (6). 16-23.

[9] B. Fry and C. Reas Processing. http://processing. org/.

[10] A. Greenfield and M. Shepard. Urban Computing and its Discontents, The Architectual League of New York, 2007.

[11] R. Harper, T. Rodden, Y. Rogers and A. Sellen (eds.). Being Human. Human-Computer Interaction in the Year 2020. Microsoft Research Ltd., 2008.

[12] E. Harris, G. Fitzpatrick, Y. Rogers, S. Price, T. Phelps and

C. Randell, From snark to park: lessons learnt moving pervasive experiences from indoors to outdoors. in Proceedings of OzCHI'04, 2004, Australian Computer Society, Inc.
[13] S. Harrison and P. Dourish, Re-Place-ing Space: The Roles of Place and Space in Collaborative Systems. . in Proceedings of CSCW'96, 1996, New York: ACM, 67 - 76.

[14] E. Hornecker, P. Marshall and Y. Rogers. From entry to access: how shareability comes about Proceedings of DPPI'07, ACM, Helsinki, Finland, 2007.

[15] L. Lessig Code: and Other Laws of Cyberspace. Basic books, 2000.

[16] J. Löwgren and E. Stolterman Thoughtful Interaction Design. MIT Press, 2004.

[17] M. McCullough Digital Ground: Architecture, Pervasive Computing and Environmental Knowing. The MIT Press, 2005.

[18] F. F. Mueller, M. R. Gibbs and F. Vetere. Design influence on social play in distributed exertion games Proceedings of CHI'09, ACM, Boston, MA, USA, 2009.

[19] K. O'Hara, M. Glancy and S. Robertshaw. Understanding collective play in an urban screen game Proceedings of CSCW'08, ACM, San Diego, CA, USA, 2008.

[20] K. O'Hara, H. Grian and J. Williams. Participation, collaboration and spectatorship in an alternate reality game Proceedings of OzCHI'08, ACM, 2008.

[21] S. Pop and M. Struppek Media Facades Festival Europe 2010. http://www.mediafacades.eu/.

[22] F. Rheinberg and Y. Manig Was macht Spaß am Graffiti-Sprayen? Eine induktive Anreizanalyse. Report Psychologie, 4. 222-234.

[23] A. F. g. Schieck, V. Kostakos and A. Penn. Exploring Digital Encounters in the Public Arena. in Shared Encounters, Springer, London, 2010.

[24] A. F. g. Schieck and E. O'Neill, The Urban Screen as a platform for communication. in Proceedings of CHI'09, 2009.

[25] F. g. Schieck, C. Briones and C. Mottram. The urban screen as a socialising platform: exploring the role of place within the urban space. in Eckardt, F. and Geelhaar, F. eds. MEDIACITY: Situations, Practices and Encounters, Frank \& Timme $\mathrm{GmbH}$, Berlin, 2008, 285 - 305.

[26] J. G. Sheridan and N. Byran-Kinns Designing for performative tangible interaction. International Journal of Arts and Technology, 1 (3-4 / 2008). 288 $-308$.

[27] M. Struppek Interactionfield. http://www. interactionfield.de/.

[28] M. N. Stukoff. Bluetooth as a Playful Public Art Interface. in Willis, K.S., Roussos, G., Chorianopoulos, K. and Struppek, M. eds. Shared Encounters, Springer, London, 2010, 127 - 151.

[29] K. S. Willis, G. Roussos, K. Chorianopulos and M. Struppek (eds.). Shared Encounters. Springer, 2010.

[30] T. V. Wolf, J. A. Rode, J. Sussman and W. A. Kellogg, Dispelling "design" as the black art of $\mathrm{CHI}$. in Proceedings of CHI'06, 2006, ACM. 